\title{
Mechanics and band gaps in hierarchical auxetic rectangular perforated composite metamaterials
}

\author{
K. Billon ${ }^{1}$, I. Zampetakis ${ }^{2,4}$, F. Scarpa ${ }^{2}$, M. Ouisse ${ }^{1 *}$, E. Sadoulet-Reboul ${ }^{1 *}$, \\ M. Collet ${ }^{3 *}$, A. Perriman ${ }^{4 *}$, A. Hetherington ${ }^{5 *}$ \\ ${ }^{1}$ Univ. Bourgogne Franche-Comté FEMTO-ST Institute CNRS/UFC/ENSMM/UTBM, \\ Department of Applied Mechanics, 24 chemin de l'Epitaphe, 25000 Besançon-FR \\ ${ }^{2}$ Advanced Composites Centre for Innovation and Science (ACCIS), University of \\ Bristol, BS8 1TR Bristol, United Kingdom \\ ${ }^{3}$ Laboratory of Tribology and Systems Dynamics (LTDS), Ecole de Centrale Lyon, 36 \\ avenue Guy de Collongue, 69134 Ecully, France \\ ${ }^{4}$ School of Cellular and Molecular Medicine, University of Bristol, BS8 1TD Bristol, \\ United Kingdom \\ ${ }^{5}$ School of Biological Sciences, University of Bristol, BS8 1QT Bristol, United Kingdom
}

\section{Abstract}

We describe in this work a composite metamaterial with a hierarchical topology made by tessellating perforations that exhibit an auxetic (negative Poisson's ratio) behaviour. We perform an analysis of the hierarchical structure by evaluating the fractal order of the topologies associated to the perforared composites. The periodic hierarchical lattice configuration shows negative Poisson's ratio characteristics at higher levels of hierarchy, even when the baseline configuration has a topology not exhibiting an auxetic behaviour. We investigate the wave propagation characteristics of these particular hierarchical lattices by using a Bloch Wave approach applied to detailed Finite Element geometries of the unit cell configurations. We show that the level of

\footnotetext{
*morvan.ouisse@femto-st.fr
} 
hierarchy creates new band gaps with large relative widths, and it also shifts the same bandgaps towards lower frequencies. We correlate the mechanical properties, fractal order and the dispersion characteristics of the multiscale auxetic perforated metamaterial with the parameters defining the geometry of the lattice and the hierarchy levels, and discuss the results in a nondimensional form to provide a performance map of the mechanical and dynamic properties.

Keywords: periodic structures, metamaterial, auxetic, vibroacoustic

\section{Introduction}

Mechanical metamaterials have been recently hailed as a new class of structural concepts able to bring novel multifunctionalities [1] by changes of compliance,shapes, or by embedding oscillators or smart materials inserts. Some examples (to name a few) are multiscale architecturally structured topologies [2], zig-zag folded sheets [3], pentamodal lattices [4], systems with distributed resonators [5, 6], smart/magnetic materials [7], tunable connectivity [8], phononic stubbed plates [9] and nonlinear dampers [10], the latter example being referred to a structure having a negative Poisson's ratio.

Negative Poisson's ratio [11] is a mechanical feature of auxetic [12] or dilational [13] materials, and indicates an unusual large volumetric deformation that corresponds to a transverse dilatation with a uniaxial tensile loading. Auxetic structures and solids have been extensively evaluated for their mechanical wave propagation behaviour, because of their strong acoustic signature and potential phononic applications $[14,15,16]$. By applying patterns of perforations it is possible to generate negative Poisson's ratio effects 
in continuum planar structures. The presence of perforations with specific geometry and spacing in a planar continuum structure creates an in-plane negative Poisson's ratio behaviour, whether one can use an elliptical [17] or rhomboidal architecture $[18,19]$. The use of perforations is quite instrumental to create hierarchical configurations by tessellating in a self-similar way the perforated pattern and obtain auxetic configurations in planar and cylindrical domains [20]. A similar approach has also been taken with the engineering of patterns of slits in fractal order [21], or following Kagome-types and various centresymmetric tessellations [22, 23]. The introduction of hierarchy in porous solids has been long recognised as a way to design enhanced specific buckling and stiffness performance [24, 25, 26, 27], as well as the transport properties of cellular and porous materials [28]. Recent work has also examined the use of cut hinges topologies in a hierarchical tessellation both from the static mechanical and in-plane wave propagation behaviour [29]. Waves in self-similar domains have several appealing features, like localization phenomena in fluid-filled periodic fractal inclusion acoustic band gap crystals or filters [30], Sierpinsky or quasi-fractal arrangements [31], and the creation of large bandgaps at lower dimensionless frequencies in beam lattices [32]. Reference [29] suggests that the general use of perforations could constitute a quite interesting strategy to design extremely tailorable bandgap materials especially at lower frequencies, due to the ease of producing these 2D metamaterials by simple automatic cutting/CNC machining.

In this work we describe a configuration of hierarchical 2D metamaterial that is produced by a self-similar generation of a rectangular perforated topology with in-plane negative Poisson's ratio ratio behaviour. The topology 
is derived by configurations identified by Sigmund when developing through Topological Optimization cellular configurations with in-plane weak shear stiffness [33]. The in-plane stiffness, negative Poisson's ratios and shear of this perforated rectangular configuration has been evaluated by Slann and co-workers both from the experimental and numerical point of view [34]. The original rectangular perforated topology maintains an in-plane auxetic behaviour, although for some specific types of pores, aspect ratios and thickness of the cut vertical side the cellular structure switches to a positive inplane Poisson's ratio behaviour. We will show that by using a hierarchical structure of this perforation we obtain a cellular $2 \mathrm{D}$ composite material that is always auxetic, even when the baseline self-similar cell is not. Quite significantly, the use of different hierarchical levels with fractal dimensions leads to tailoring and enlarging full and partial bandgaps in a way that could be used to design 2D metamaterials with multiple filtering capabilities.

\section{Geometry of the hierarchical perforated auxetic lattice}

The fundamental unit cell of the perforated lattice is shown in Figure 2. The rectangular perforation is described by the parameters $S, a$ and $b$, which

represent the width of the vertical rib, and the length and vertical thickness of the perforation respectively. The overall width of the cell is $r=a+b+2 S$ and the aspect ratio $A R$ is defined as $a / b$. The unit cell has a double symmetry around the central horizontal and vertical axis. The different hierarchical levels of the perforated structure are produced by repeating the fundamental unit cell on each quarter unit as shown in Figure 2. For a given aspect ratio of the perforation the equivalent volume fraction $(\phi)$ of the cellular structure 
(defined as the ratio between the region on the unit filled with material and the total surface of the unit cell 2) varies significantly with the vertical rib $S$ parameter. At low $S$ levels the volume fraction tends to increase even more between the first and second hierarchical levels, with an increase for example of 1.48 times between levels 1 and levels 2 of the unit at $A R=a / b=4$. The increment of the $S$ parameter for a given hierarchical level is also significant, with a $140 \%$ decrease of the volume fraction between $S=0.2$ and $S=0.8$. It is worth of notice that for the purpose of this work the volume fraction is directly proportional to the relative density $\rho / \rho_{c}$, in which $\rho$ and $\rho_{c}$ are the densities of the cellular structure and the solid material constituting the structure itself, respectively.

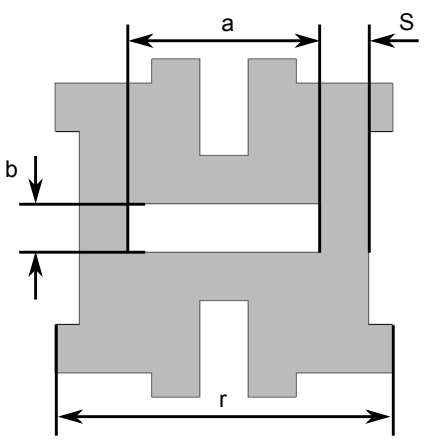

(a)

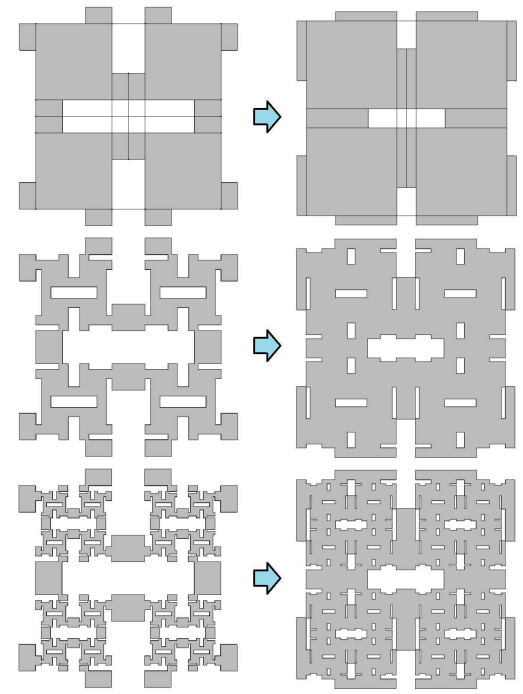

(b)

Figure 1: a) Geometry parameters of the base unit cell. b) Hierarchical, auxetic rectangular perforations at Level 1,2 and 3 with $A R=4$ and from $S=0.2$ to $S=0.8$. 
The hierarchical tessellation repeats itself only in the central quarters of the cell belonging to the previous level (Figure 2). As it will be evident from the analysis of the in-plane mechanical properties, for a constant aspect ratio the variation of the spacing parameter $S$ may lead to a topology with discontinuous tessellation because of the particular strategy chosen for the construction of the hierarchy. We have therefore analysed the fractal order of the topologies at different hierarchical levels to assess the degree of selfsimilarity in the hierarchical perforated lattice. One of the most commonly accepted fractal dimension estimate is the Hausdorff-Besicovitch dimension $D$, which is defined as the logarithmic ratio between the number $N$ of the internal homotheties of an object and the reciprocal of the common ratio $R$ of this homothety [35]:

$$
D=\frac{\log N}{\log r},
$$

where $r=1 / R$. We have used in this analysis one of the most popular techniques to estimate the fractal dimension, the box-counting approach [35, 36]. In this case the common homotheteis consists in boxes of size $R$. Our algorithmic analysis was initially performed with the images converted into a binary format, via thresholding, where pixels with value 0 correspond to the background and with value 1 to the object of interest, in order to be successfully implemented for the box-counting algorithm [35, 37]. The box counting algorithm applied on the binary images [38] initially ensured that the images had an even number of pixels via adding background (0s) pixels. Boxes of various size $R$ were overlaid on each image and the boxes that included object pixels (1s) were counted, so the number $N$ of such boxes, at 
each size $R$, was determined. The slope obtained from the linear regression of the resulting plot of $\log N$ versus $\log (1 / R)$ corresponded to the fractal dimension value for each structure. The analysis of the fractal dimension of the lattices has been carried out for the three different levels and varying aspect ratios between 3 and 6 . The $S$ spacing has been varied between 0.2 and 0.8 .

Figure 3 shows the results of this parametric analysis. At Level 1 the fractal order varies between 1.72 and 1.65, with the lowest values at the lower end of the parametric scale for $A R$ and $S$. When the order of the hierarchy is increased to 2 the fractal dimension tends to decrease on average by $5 \%$, with the lowest values corresponding again to the lowest combinations of $A R$ and $S$. The difference of the fractal dimension between levels 2 and 3 is however small, on average less than $1 \%$. It is worth noticing that for high aspect ratios and spacing values the three hierarchical levels tend to provide quite similar fractal dimensions (between 1.73 and 1.70). The closeness of the fractal orders associated to the second and third hierarchy levels suggests a potential similarity in their in-plane mechanical properties, and a difference with the ones belonging to Level 1. As it will be evident in the next paragraph, hierarchy levels higher than 1 do actually exhibit a set of in-plane stiffness and Poisson's ratios values that difference them from the original baseline rectangular perforation.

\section{3. in-plane mechanics}

The in-plane mechanical properties have been computed using a Finite Element approach applied to a quarter unit cell. The FE models have been 


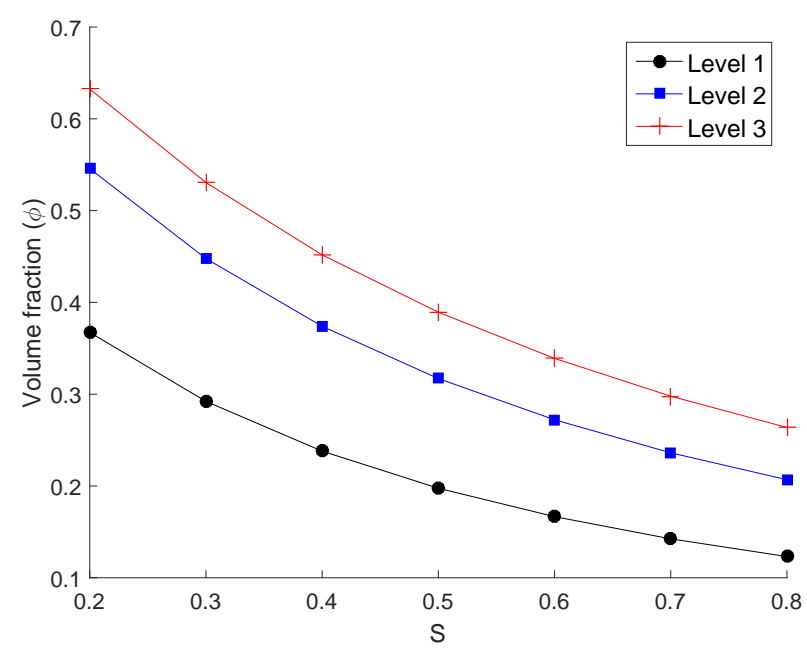

Figure 2: Volume fraction for various sizes of the intercell spacing S at Levels 1, 2 and 3.

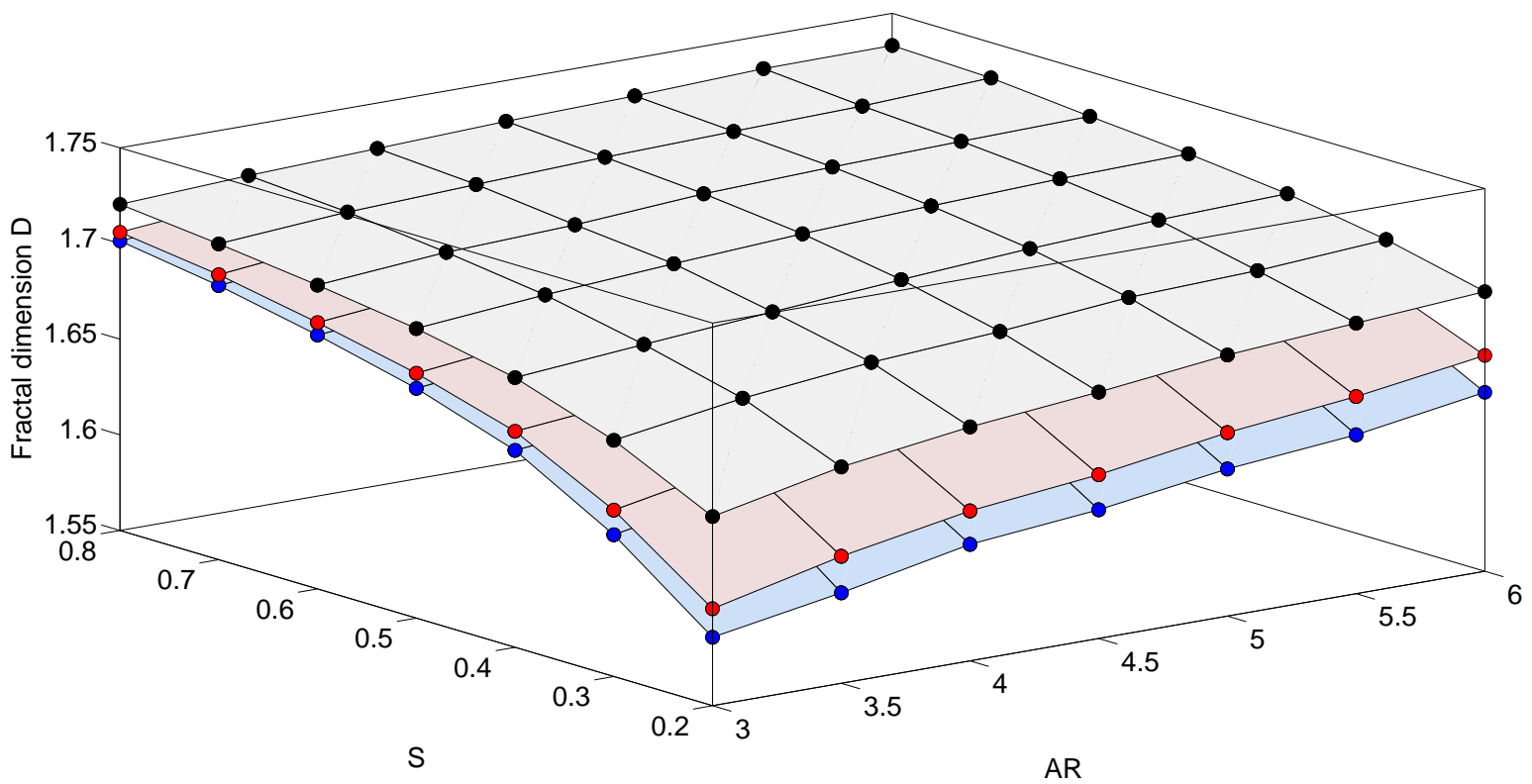

Figure 3: Distribution of the fractal order of the hierarchical tessellations at different levels for varying aspect and spacing ratios. ० Level 1; ○ Level 2; ○ Level 3. 
developed using the commerical code ANSYS Rel. 14.0. The elements used were 8-nodes serendipidity PLANE82 with two translational degrees of freedom in plane stress. After a convergence test the mesh density was set at four elements per linear length of the parameter $S(S / 4)$. The same discretisation was also maintained for the hierarchical levels. The in-plane Young's moduli $E_{1}$ and $E_{2}$ have been predicted by applying sliding conditions on the external side of the quarter unit cell, an imposed uniform displacement along the direction of the mechanical loading, while nodes of the elements belonging to the non-loaded side were coupled along the transverse direction [39]. The uniaxial Young's moduli were calculated by averaging the reaction forces along the line subjected to the uniform distribution, calculating the resulting stress and dividing it by the corresponding imposed strain. The in-plane Poisson's ratio $\nu_{12}$ and $\nu_{21}$ were computed first by calculating the transverse strain from the coupled transversal deformation under the uniaxial loading, and then using the classical definition of Poisson's ratio $\nu_{i j}=-\epsilon_{j} / \epsilon_{i}$, in which $\epsilon_{i}$ is the imposed uniaxial strain along the $i$-direction and $\epsilon_{j}$ is the resulting homogenised transverse one. The in-plane shear modulus $G_{12}$ was calculated starting from a quarter unit cell with support boundary conditions, but this time being subjected on the external sides to combined tensile and compressive uniaxial strains representing a simple shear loading [40] (figure 4).

The results have been initially benchmarked against experimental data on the in-plane rectangular perforations described in [34], and we obtained discrepancies between $1 \%$ and $2 \%$ with the in-plane Young's moduli and Poisson's ratios of perforated plates. All the numerical results have been 
nondimensionalised against the Young's modulus $E_{c}$ of the solid material and the relative density $\rho / \rho_{c}$ (i.e., the volume fraction described above).

Figures $5 \mathrm{a}, 5 \mathrm{~b}, 5 \mathrm{c}$ and $5 \mathrm{~d}$ show the behaviour of the normalised engineering constants for a constant aspect ratio $A R=4$ and varying parameter $S$. The perforated structure at the first hierarchical level (1) is isotropic, but the introduction of different levels of hierarchy induces the presence of a special orthotropic configuration $\left(E_{1} \nu_{21}=E_{2} \nu_{12}\right)$ due to the particular shape at level 1 repeated with the scale ratio. The Poisson's ratio of the level 1 configuration is not always auxetic, and for large values of $S$ tends to have a zero or marginally positive Poisson's ratio (Figure 5d). At higher hierarchical levels the cellular configuration becomes however auxetic for all the configurations considered. The introduction of nested perforated cells increases significantly the coupling between the uniaxial loading and the rotation, and therefore creates the in-plane negative Poisson's ratio effect typical of structural chiral materials, and more in general of micropolar solids $[41,42]$. The normalised in-plane stiffness $\left(E_{1} / E_{c}\right) /\left(\rho / \rho_{c}\right)$ decreases by almost 2 orders of magnitude at low $S$ values between levels 1 and 3 . That decrease is lower for $S \rightarrow 0.8$, albeit still at more than one order of magnitude (Figure 5a). A similar trend can also be observed in the case of $\left(E_{2} / E_{c}\right) /\left(\rho / \rho_{c}\right)$, although this normalised stiffness does not appear to show the same sensitivity versus the rib width; at higher values of $S$ the in-plane stiffness between the different levels shows a very little difference (Figure 5b).

Jumps in the mechanical properties are observed at Levels 2 and 3 due to transition of parts of the subunits from contact to noncontact between the voids. For a constant aspect ratio the variation of the spacing parameter 
$S$ may lead to a topology with discontinuous tessellation because of the particular strategy chosen for the construction of the hierarchy (Figures 5).

Figures $6 \mathrm{a}, 6 \mathrm{~b}, 6 \mathrm{c}$ and $6 \mathrm{~d}$ show the behaviour of the normalised engineering constants for a constant aspect ratio $S=0.2$ and varying parameter $A R$. At level 1 and higher hierarchical levels the cellular configuration are always auxetic for all the configurations considered (Figure 6d). The normalised in-plane stiffness $\left(E_{1} / E_{c}\right) /\left(\rho / \rho_{c}\right)$ decreases by almost 2 orders of magnitude from $A R=3$ to $A R=5.5$ values between levels 1 and 3 , and that decrease is higher for $A R \rightarrow 6$ (Figure 6a). A similar trend can also be observed in the case of $\left(E_{2} / E_{c}\right) /\left(\rho / \rho_{c}\right)$, although this normalised stiffness does not appear to show the same sensitivity versus the rib width, and at higher values of $A R$ the in-plane stiffness between the different levels stays constant (Figure 6b).

\section{Wave propagation}

Understanding the behavior of the perforated composite metamaterial in an extended frequency range requires a finer description of the geometry, and this is particularly important for the particular hierarchical metamaterial configuration evaluated in this work. The wave propagation analysis related to a fully detailed geometry model has been carried out by applying the Floquet-Bloch method [43]. To this end a plain stress Finite Element mesh has been created using the COMSOL platform. The models for all levels were composed by triangular elements (quadratic interpolation), with varying mesh size based on the level of the topology. As an example, for a configuration of $A R=4$ and $S=0.2$ a Level 1 model has 506 elements that were increasing to 1770 and 7944 when passing to Levels 2 and 3 respectively. 
According to Floquet-Bloch theorem, the boundary conditions applied along the 1 and 2 directions at the cell edges can be represented as $u_{R}=e^{-j k_{x} r} u_{L}$ and $v_{R}=e^{-j k_{2} r} v_{L}$, where $u_{R}$ (resp. $v_{R}$ ) is the displacement on the right edge and $u_{L}$ (resp. $\left.v_{L}\right)$ is the displacement on the left edge in $x$ (resp. $y$ ) directions, $k_{x}$ and $k_{y}$ are respectively the wavenumbers in the $x$ and $y$ directions. The equations of motion of the system assuming an harmonic solution can be described as:

$$
\rho \omega^{2} u+\nabla \sigma=0, \sigma=C: \epsilon, u_{R}=e^{-j k_{x} r} u_{L}, v_{R}=e^{-j k_{2} r} v_{L}
$$

where $u \exists \mathbb{R}^{2}$ and $\sigma, C$ and $\epsilon$ are the stress, second order elastic and strain tensors respectively. The associated undamped eigenvalue problem of Equation (2) with the Floquet-Bloch wave transformation has been solved with the Pardiso solver. The eigenvalue problem is parametrised against the wavenumbers $k_{1}$ and $k_{2} \in[0, \pi / r]$. The dispersion curves are plotted on the contour of the first Brillouin zone (k-space). The frequencies identifying the bandgaps can be found by considering the contour of the irreducible Brillouin zone for regular systems [44]. However, although this approach is extensively used in open literature, no formal proof of its validity is given, and therefore the results obtained need to be treated with care [45]. To compare the results of the eigenvalue analysis at different levels we have computed for each geometry configuration of the perforated composite plate the equivalent volume fraction $(\phi)$ and computed by Finite Element the natural frequency of a rectangular plate (plane strain) with the same overall dimension of the lattice with Poisson's ratio equal to the one of the core material $\left(\nu_{c}\right)$, scaled density $\bar{\rho}=\phi \rho_{c}$ and equivalent Young's modulus $\bar{E}=E_{c} \phi^{2}[46]$. The resulting 
fundamental frequency is denominated as $\omega_{p}$.

The dispersion curves in the k-space of the lattices at Level 1 for the case of $A R=4, S=0.3$ are shown in Figure 7a. One can observe the presence of partial bandgaps in several sections of the irreducible Brillouin zone and nondimensional frequencies, however a sizable bandgap across the whole $\mathrm{k}$ space exists around $\omega / \omega_{p}=3$. At the next level the number of complete bandgaps increases, the bandgap that exists at Level 1 appears to narrow and decreases to $\omega / \omega_{p} \approx 2.2$ and - quite significantly - narrower complete bandgaps appear at lower frequencies (Figure 7b). Level 3 witnesses a significant increase of the number of bandgaps at even lower normalised frequencies (down to $\omega / \omega_{p} \approx 0.3$ ), and the presence of a very large bandgap centered around $\omega / \omega_{p}=3.8$ (Figure 7c).

It is interesting to observe the total width of the bandgaps existing at the different levels as a percentage of the nondimensional frequency space up to $\omega / \omega_{p}=5$ for an aspect ratio of 4 (Figure 8 ). The total width tends to decrease for increasing values of the spacing $S$, and this feature is common to all the lattice levels considered. At $S=0.2$ the total band gap size increases from $13 \%$ to $16 \%$ when moving from Level 1 to Level 2, and to a quite significant $42 \%$ when passing at Level 3 . From increasing values of the parameter $S$ the total size of the bandgaps at level 1 decreases significantly, and from $S=0.4$ onwards no bandgaps are present in the lattice at that level. The decrease of the total bandgap width versus the spacing of the lattice is also present in the perforations at Levels 2 and 3, although the latter still show almost the same percentage of bandgap size at $S=0.8$ that Level 1 exhibits for $S=0.4$. 
For the same aspect ratio of perforations the distribution of the bandgaps and their width according to the $S$-parameter varies in a significant manner. A single bandgap is present for $S=0.2, S=0.3$ and $S=0.4$ at Level 1, but in the latter case the bandgap becomes narrower and centered at a higher $\omega / \omega_{p}$ value (Figure 9a). At Level 2 between $S=0.2$ and $S=0.5$ there is an evident shift of the central frequencies of the bandgaps at higher nondimensional frequencies, however the number of bandgaps decreases thereafter (Figure $9 \mathrm{~b})$. The sudden drop in number and size of the bandgaps is due to the change of morphology and connectivity that the perforated lattice exhibits (see Figure 5a). A similar trend can also be observed at level 3 moreover the density of bandgaps increases significantly (Figure 9c).

The Bloch wave simulations are related to periodic unit cells with the detailed geometry associated to the hierarchy of the microstructure. It is interesting at this point to investigate the correlation between the dispersion properties calculated from asymptotic homogenized mechanical properties and the high-fidelity predictions provided by the Bloch wave approach [47]. A plate with the same overall dimensions of the high-fidelity using cell (triangular elements with a mesh density of 100 elements per side cell) has been modelled with the homogenised mechanical properties described in Paragraph III and the analogous eigenvalue problem (2) with Bloch wave conditions has been solved by parametrising against the wavenumbers $k_{1}$ and $k_{2}$. Examples of these simulations are shown in Figure ??, in which the comparison with the high-fidelity full-scale Bloch wave model is performed for $A R=4$ and $S=0.2$. Also in this case, the results have been nondimensionalised against the natural frequency $\omega_{p}$. At Level 1 (??a) its is possible 
to observe that the homogenization approach provides a close comparison with the full-scale high-fidelity Bloch wave model. The first dispersive curve shows an a very good agreement with the analogous high-fidelity geometry over the whole k-s space. The second dispersive curve shows a general overestimation of the nondimensional frequency with the full-geometry simulation within the $\Gamma-\mathrm{X}$ and $\mathrm{M}-\Gamma$ zones. In the central $\mathrm{k}$-space sector $\mathrm{X}-\mathrm{M}$ the second homogenized dispersive curve tends to follow and overestimate the analogous third curve. A similar trend, this time with a more conservative behaviour compared to the real geometry case is the one observed for Level 2 (Figure ??b). In this case the two homogenized dispersive curves have higher values of $\omega / \omega_{p}$ over the whole k-s space. It also appears that the dispersion curves from the homogenized models tend to converge to the full-scale geometric detailed one towards the end of the $\mathrm{M}-\Gamma$ sector. It is remarkable to notice how similar is the trend of the homogenized dispersion curves in Level 3 compared to the ones of Level 2 (Figure ??c), with both overestimating the nondimensional frequencies provided by the high-fidelity Bloch wave model, and following the trends of the first two dispersive curves of the detailed geometric model within the ends of the $\Gamma-\mathrm{X}$ and $\mathrm{M}-\Gamma$ sectors of the k-s space. The rich wave modal density of the hierarchical perforated configurations cannot be however fully captured by the homogenized dispersions relations calculated with the Christoffel's equations. Even at low nondimensional frequency values and for the lowest hierarchical level one can observe the presence of dispersive branches and local band gaps created by the fractal-type topology that the asymptotic homogenization is not able to reproduce. 


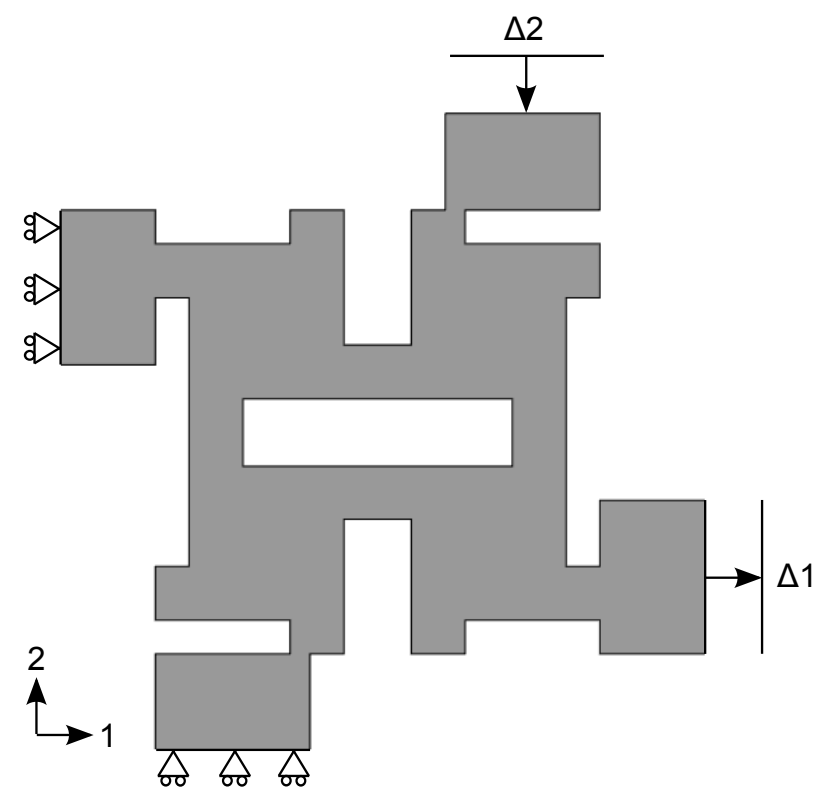

Figure 4: Boundary conditions for virtual biaxial shear-test at Level 2. 


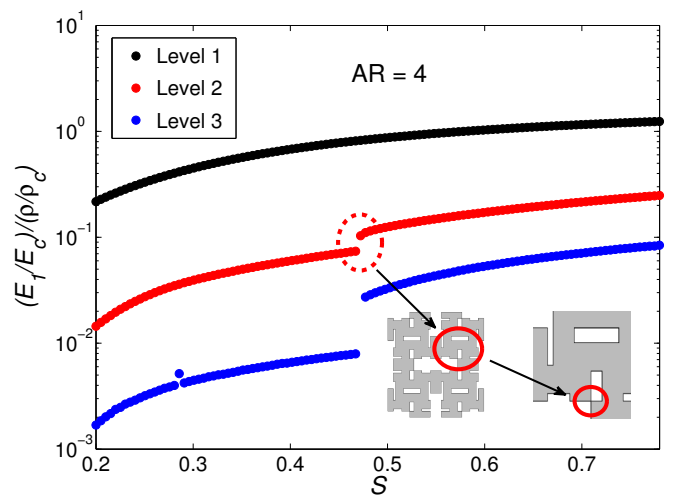

(a)

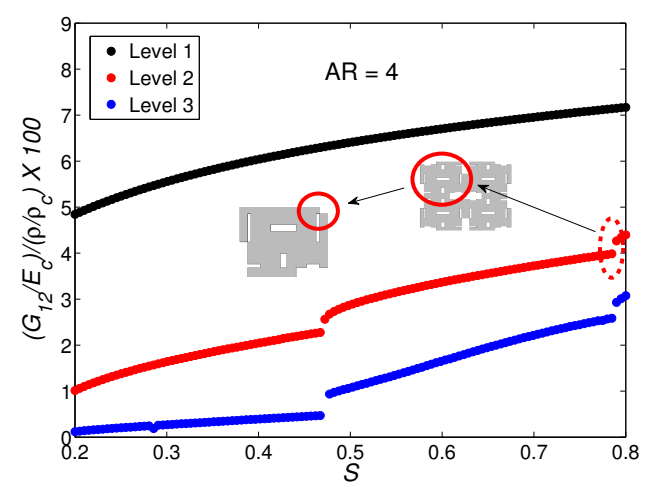

(c)

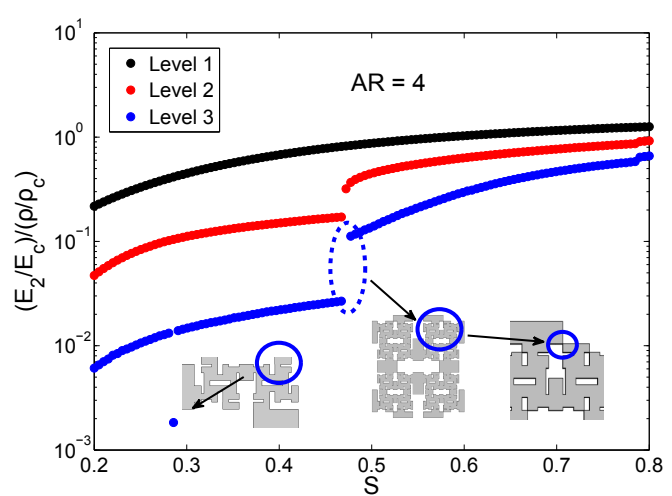

(b)

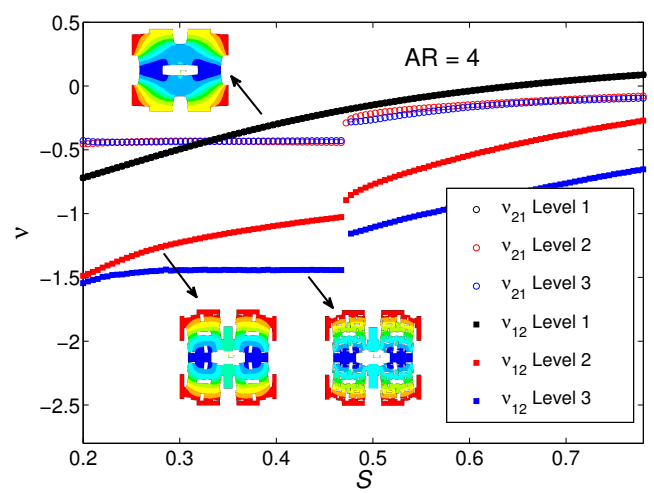

(d)

Figure 5: Variation of the nondimensional Young's moduli a) $E_{1}$, b) $E_{2}$, c) in-plane shear modulus $G_{12}$ and d) in-plane Poison's ratios $\nu_{12}$ and $\nu_{21}$ versus the intercell spacing $S$ at Levels 1,2 and 3 for an aspect ratio of 4 . 


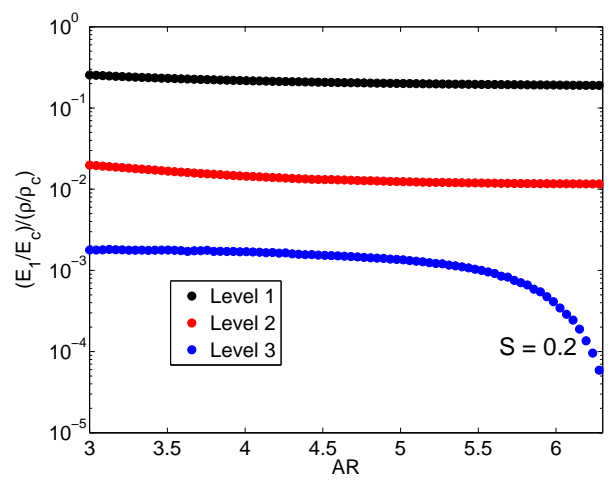

(a)

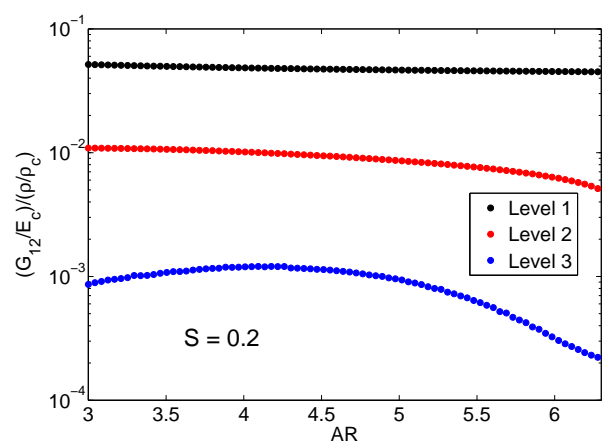

(c)

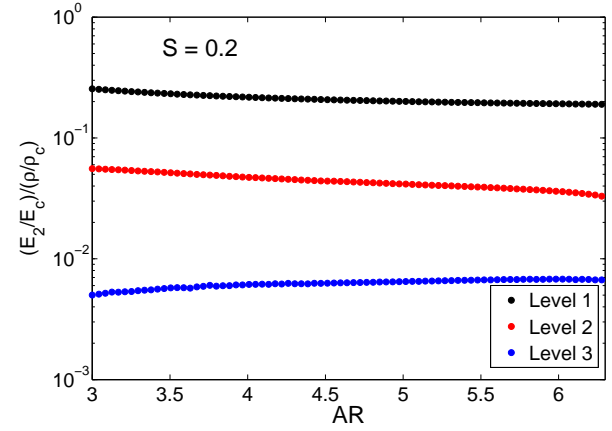

(b)

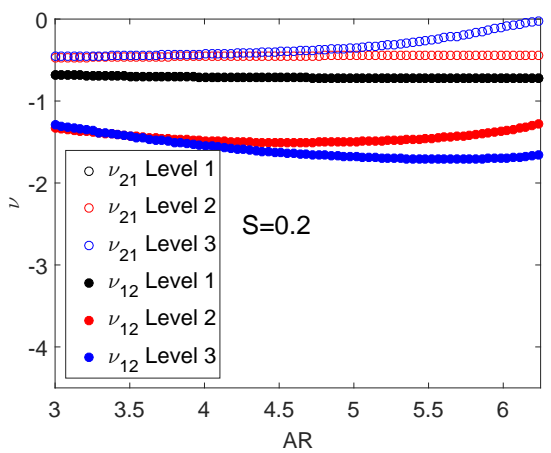

(d)

Figure 6: Parametric analysis of the nondimensional Young's moduli a) $E_{1}$, b) $E_{2}$, c) shear modulus $G_{12}$ and d) in-plane Poisson's ratios for varying aspect ratios at the different hierarchical levels. The $S$ parameter is constant at 0.2 . 


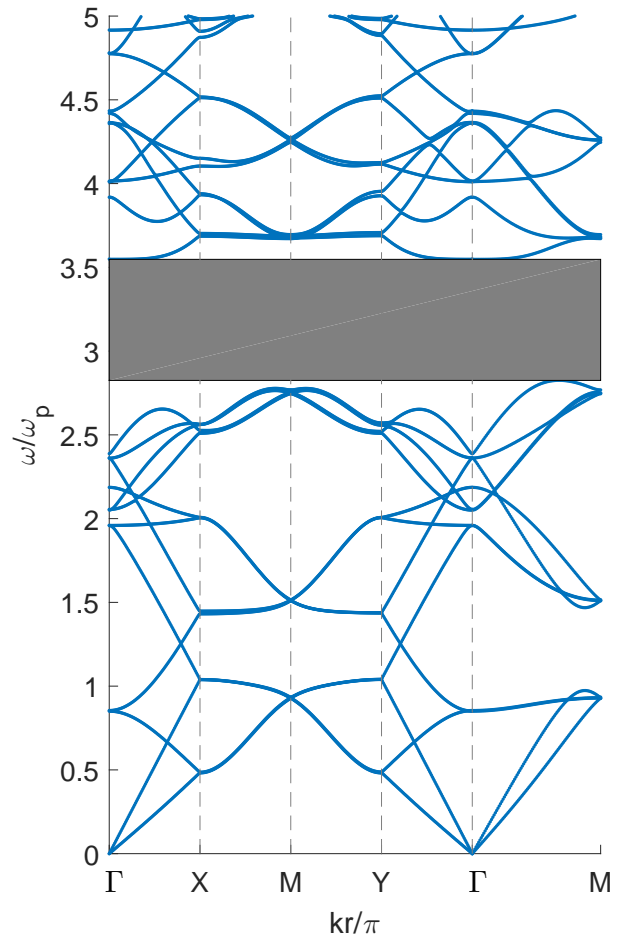

(a)

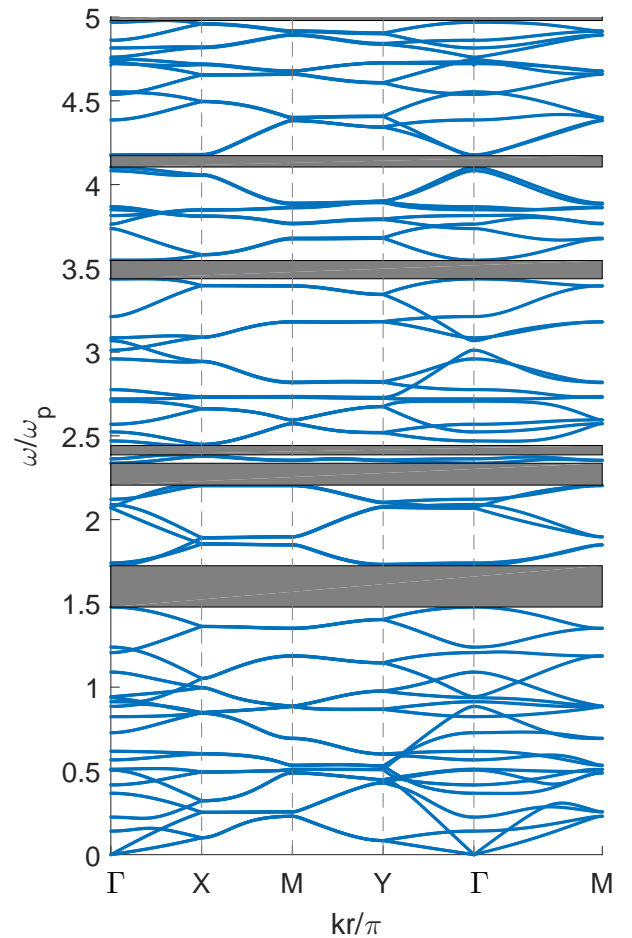

(b)

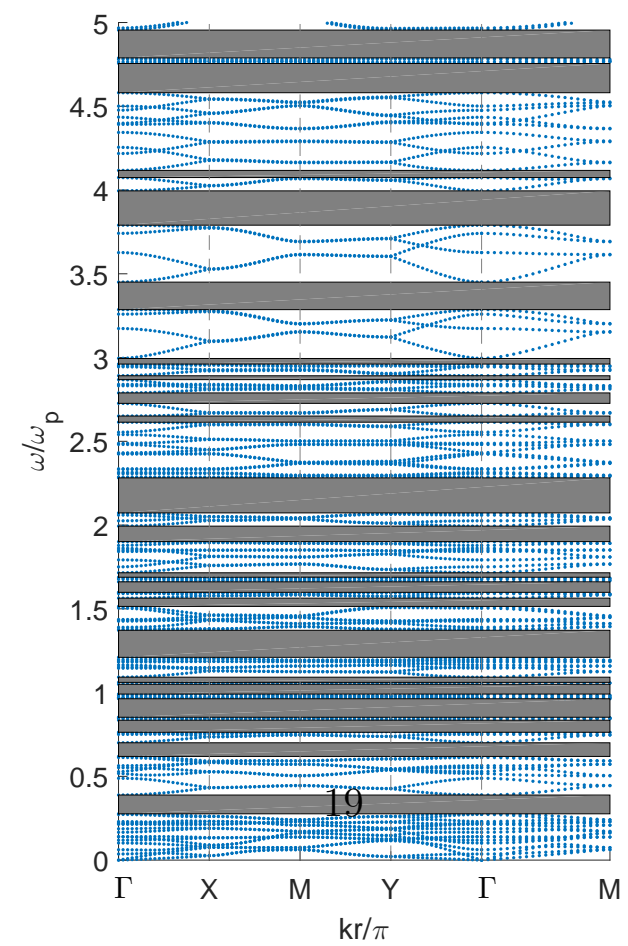

(c) 


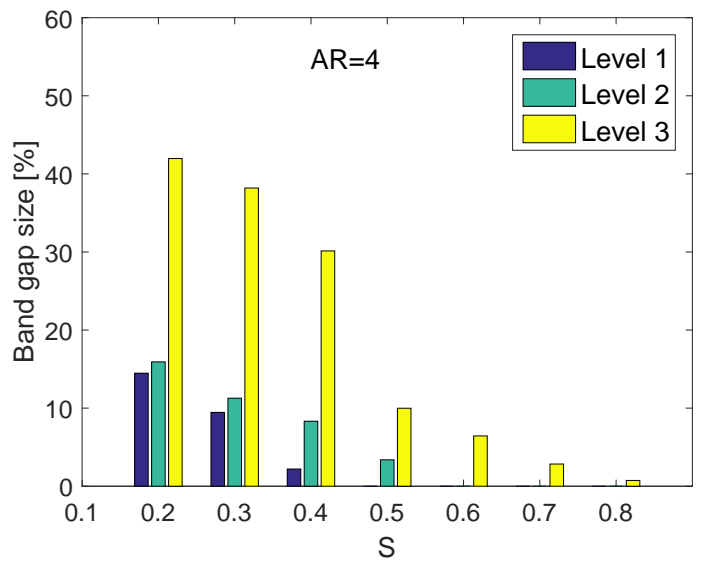

Figure 8: Total width of the band gaps for various intercell spacing $S$ at Levels 1, 2 and 3. 


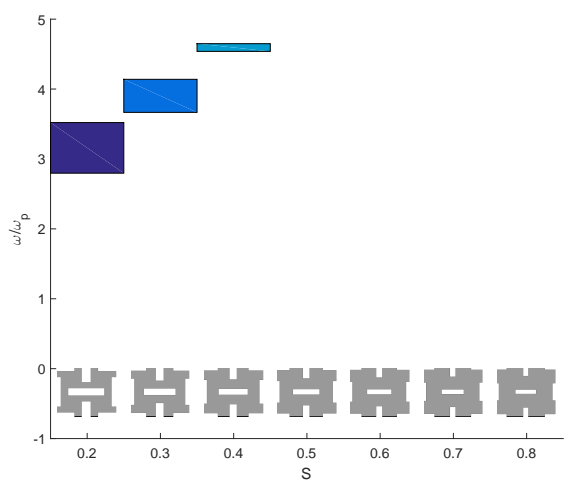

(a)

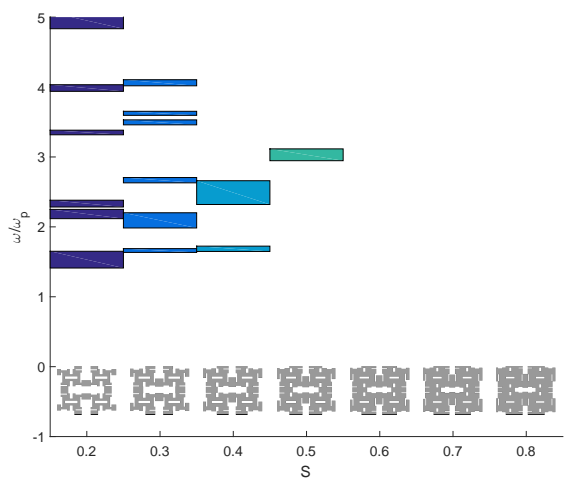

(b)

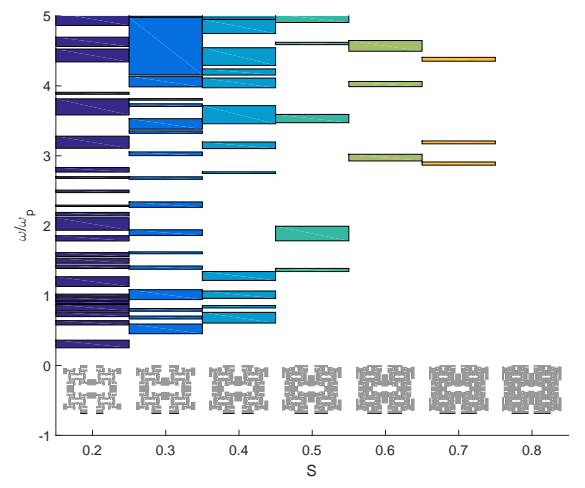

(c)

Figure 9: Band gap distribution versus different $S$-parameter configurations for a) level 1, b) level 2 and c) level 3 . 


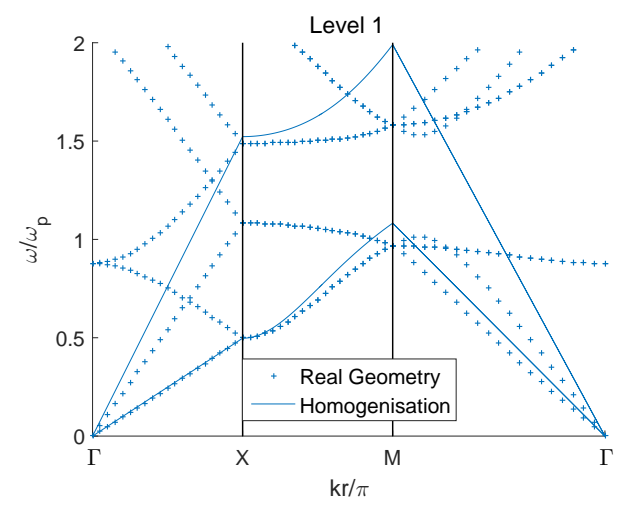

(a)

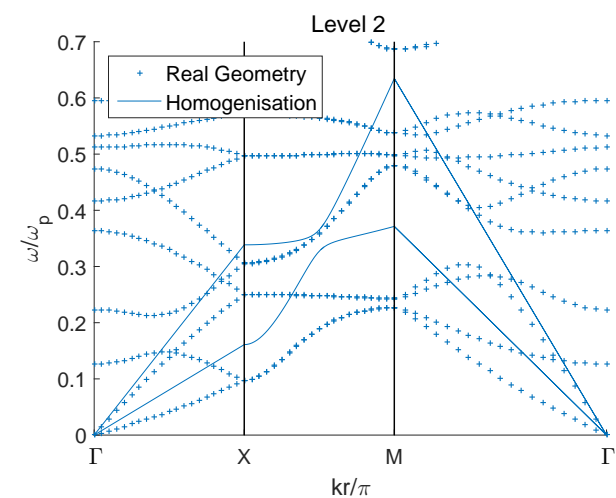

(b)

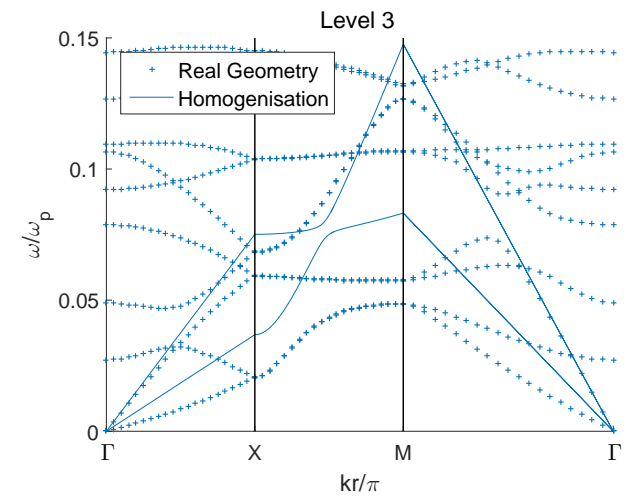

(c)

Figure 10: Comparison between homogenized and full-scale Bloch wave dispersions of the hierarchical lattices for $A R=4$ Aand $S=0.2$ a) level 1, b) level 2, c) level 3 


\section{Conclusion}

We have presented a hierarchical configuration of a rectangular perforation pattern that allows to create auxetic deformation behaviors in a $2 \mathrm{D}$ material composite planar structure. The use of the hierarchical configuration proposed allows to generate a negative Poisson's ratio and controlled orthotropy from a baseline perforated unit that not necessarily is auxetic, but it is always isotropic. The hierarchical construction leads to fractal dimensions of the lattices that tend to vary noticeably between the first and the second level, and with lower differences between the second and third self-similar constructions. The hierarchical rectangular perforated topology proposed is able to create a very significant broad range of full bandgaps and tailored centre frequencies based on the levels of hierarchy and geometry parameters of the self-similar cells. Moreover, it offers the opportunity to engineer band gaps by applying simple perforations and cuts topologies in planar composite structures that could be made with arbitraty material substrates.

\section{Acknowledgment}

This work was financed by The French National Research Agency under grant number ANR-12-JS09-008-COVIA. It has been performed in cooperation with the Labex ACTION program (ANR-11-LABX-0001-01). The Authors also acknowledge the support of the FP7-AAT-2012-RTD-L0-341509 MorphElle project for providing part of the computational tools used in this work and support to KB's stay at the University of Bristol. IZ acknowledges 
the support of Dstl and the EPSRC Centre for Doctoral Training in Synthetic Biology (SynBio).

[1] M. Kadic, T. Bückmann, R. Schittny, M. Wegener, Metamaterials beyond electromagnetism, Reports on Progress in Physics 76 (12) (2013) 126501.

URL http://stacks.iop.org/0034-4885/76/i=12/a=126501

[2] J.-H. Lee, J. P. Singer, E. L. Thomas, Micro-/nanostructured mechanical metamaterials, Advanced Materials 24 (36) (2012) 4782-4810. doi:10.1002/adma.201201644.

URL http://dx.doi.org/10.1002/adma. 201201644

[3] M. Eidini, G. H. Paulino, Unraveling metamaterial properties in zigzag-base folded sheets, Science Advances 1 (8). arXiv:http://advances.sciencemag.org/content/1/8/e1500224.full.pdf, doi:10.1126/sciadv.1500224.

URL http://advances.sciencemag.org/content/1/8/e1500224

[4] M. Kadic, T. Bückmann, R. Schittny, M. Wegener, On anisotropic versions of three-dimensional pentamode metamaterials, New Journal of Physics 15 (2) (2013) 023029.

URL http://stacks.iop.org/1367-2630/15/i=2/a=023029

[5] H. H. Huang, C. T. Sun, Wave attenuation mechanism in an acoustic metamaterial with negative effective mass density, New Journal of Physics 11 (1) (2009) 013003.

URL http://stacks.iop.org/1367-2630/11/i=1/a=013003 
[6] M. Nouh, O. Aldraihem, A. Baz, Wave propagation in metamaterial plates with periodic local resonances, Journal of Sound and Vibration 341 (0) (2015) 53 - 73. doi:http://dx.doi.org/10.1016/j.jsv.2014.12.030. URL http://www. sciencedirect.com/science/article/pii/S0022460X14010347

[7] J. N. Grima, R. Caruana-Gauci, M. R. Dudek, K. W. Wojciechowski, R. Gatt, Smart metamaterials with tunable auxetic and other properties, Smart Materials and Structures 22 (8) (2013) 084016.

URL http://stacks. iop.org/0964-1726/22/i=8/a=084016

[8] P. Wang, F. Casadei, S. H. Kang, K. Bertoldi, Locally resonant band gaps in periodic beam lattices by tuning connectivity, Phys. Rev. B 91 (2015) 020103. doi:10.1103/PhysRevB.91.020103.

URL http://link.aps.org/doi/10.1103/PhysRevB.91.020103

[9] M. Oudich, M. Senesi, M. B. Assouar, M. Ruzenne, J.-H. Sun, B. Vincent, Z. Hou, T.-T. Wu, Experimental evidence of locally resonant sonic band gap in two-dimensional phononic stubbed plates, Phys. Rev. B 84 (2011) 165136. doi:10.1103/PhysRevB.84.165136.

URL http://link.aps.org/doi/10.1103/PhysRevB.84.165136

[10] Y. Ma, F. Scarpa, D. Zhang, B. Zhu, L. Chen, J. Hong, A nonlinear auxetic structural vibration damper with metal rubber particles, Smart Materials and Structures 22 (8) (2013) 084012.

URL http://stacks.iop.org/0964-1726/22/i=8/a=084012

[11] R. Lakes, Foam structures with a negative poisson's ratio, Science 235 (4792) (1987) 1038-1040. doi:10.1126/science.235.4792.1038. 
[12] K. E. Evans, A. Alderson, Auxetic materials: Functional materials and structures from lateral thinking!, Advanced Materials 12 (9) (2000) 617-628. doi:10.1002/(SICI)1521-4095(200005)12:9;617::AIDADMA617¿3.0.CO;2-3.

[13] T. Bückmann, R. Schittny, M. Thiel, M. Kadic, G. W. Milton, M. Wegener, On three-dimensional dilational elastic metamaterials, New Journal of Physics 16 (3) (2014) 033032.

URL http://stacks.iop.org/1367-2630/16/i=3/a=033032

[14] M. Ruzzene, F. Scarpa, Directional and band-gap behavior of periodic auxetic lattices, physica status solidi (b) 242 (3) (2005) 665-680. doi:10.1002/pssb.200460385.

[15] A. Spadoni, M. Ruzzene, Structural and acoustic behavior of chiral truss-core beams, Journal of Vibration and Acoustics 128 (2006) 616626.

[16] A. Sparavigna, Phonons in conventional and auxetic honeycomb lattices, Phys. Rev. B 76 (2007) 134302. doi:10.1103/PhysRevB.76.134302.

URL http://link.aps.org/doi/10.1103/PhysRevB.76.134302

[17] K. Bertoldi, P. M. Reis, S. Willshaw, T. Mullin, Negative Poisson's ratio behavior induced by an elastic instability., Advanced materials (Deerfield Beach, Fla.) 22 (3) (2010) 361-6. doi:10.1002/adma.200901956.

URL http://www.ncbi.nlm.nih.gov/pubmed/20217719

[18] J. Grima, K. Evans, Auxetic behavior from rotating squares, 
Journal of Materials Science Letters 19 (17) (2000) 1563-1565. doi:10.1023/A:1006781224002.

[19] J. N. Grima, R. Gatt, Perforated Sheets Exhibiting Negative Poisson's Ratios, Advanced Engineering Materials 12 (6) (2010) 460-464. doi:10.1002/adem.201000005.

URL http://doi.wiley.com/10.1002/adem.201000005

[20] R. Gatt, L. Mizzi, J. Azzopardi, K. Azzopardi, D. Attard, A. Casha, J. Briffa, J. Grima, Hierarchical auxetic mechanical metamaterials, Scientific reports 5 .

[21] Y. Cho, J.-H. Shin, A. Costa, T. A. Kim, V. Kunin, J. Li, S. Y. Lee, S. Yang, H. N. Han, I.-S. Choi, D. J. Srolovitz, Engineering the shape and structure of materials by fractal cut, Proceedings of the National Academy of Sciences 111 (49) (2014) 1739017395. arXiv:http://www.pnas.org/content/111/49/17390.full.pdf, doi:10.1073/pnas.1417276111.

URL http://www. pnas.org/content/111/49/17390. abstract

[22] S. Shan, S. H. Kang, Z. Zhao, L. Fang, K. Bertoldi, Design of planar isotropic negative poisson's ratio structures, Extreme Mechanics Letters 4 (2015) 96 - 102. doi:http://dx.doi.org/10.1016/j.eml.2015.05.002. URL http://www. sciencedirect.com/science/article/pii/S2352431615000759

[23] L. Mizzi, K. M. Azzopardi, D. Attard, J. N. Grima, R. Gatt, Auxetic metamaterials exhibiting giant negative poisson's ratios, physica status solidi (RRL) - Rapid Research Letters 9 (7) (2015) 425-430. 
doi:10.1002/pssr.201510178.

URL http://dx.doi.org/10.1002/pssr. 201510178

[24] R. Lakes, Materials with structural hierarchy, Nature 361 (6412) (1993) $511-515$.

URL http://dx.doi .org/10.1038/361511a0

[25] C. Taylor, C. Smith, W. Miller, K. Evans, The effects of hierarchy on the in-plane elastic properties of honeycombs, International Journal of Solids and Structures 48 (9) (2011) 1330 - 1339. doi:http://dx.doi.org/10.1016/j.ijsolstr.2011.01.017.

URL http://www.sciencedirect.com/science/article/pii/S0020768311000266

[26] Y. Sun, N. M. Pugno, In plane stiffness of multifunctional hierarchical honeycombs with negative poisson's ratio substructures, Composite Structures 106 (2013) $681-689$. doi:http://dx.doi.org/10.1016/j.compstruct.2013.05.008.

URL http://www.sciencedirect.com/science/article/pii/S0263822313002183

[27] Y. Sun, B. Wang, N. Pugno, B. Wang, Q. Ding, Inplane stiffness of the anisotropic multifunctional hierarchical honeycombs, Composite Structures 131 (2015) 616 - 624. doi:http://dx.doi.org/10.1016/j.compstruct.2015.06.020. URL http://www.sciencedirect.com/science/article/pii/S0263822315004833

[28] Y. Sun, Q. Chen, N. Pugno, Elastic and transport properties of the tailorable multifunctional hierarchical honeycombs, Composite Structures $107 \quad$ (2014) $698 \quad-\quad 710$. 
doi:http://dx.doi.org/10.1016/j.compstruct.2013.07.012.

URL http://www.sciencedirect.com/science/article/pii/S0263822313003358

[29] Y. Tang, G. Lin, L. Han, S. Qiu, S. Yang, J. Yin, Design of hierarchically cut hinges for highly stretchable and reconfigurable metamaterials with enhanced strength, Advanced Materials 27 (44) (2015) 7181-7190. doi:10.1002/adma.201502559.

URL http://dx.doi.org/10.1002/adma. 201502559

[30] R. C. Norris, J. S. Hamel, P. Nadeau, Phononic band gap crystals with periodic fractal inclusions: Theoretical study using numerical analysis, Journal of Applied Physics 103 (10). doi:http://dx.doi.org/10.1063/1.2931955.

URL http://scitation.aip.org/content/aip/journal/jap/103/10/10.1063/1.2931955

[31] S. Castiñeira-Ibáñez, C. Rubio, J. Redondo, J. V. Sánchez-Pérez, Quantitative characterization of bandgap properties of sets of isolated acoustic scatterers arranged using fractal geometries, Applied Physics Express 7 (2014) 042201.

[32] Q. J. Lim, P. Wang, S. J. A. Koh, E. H. Khoo, K. Bertoldi, Wave propagation in fractal-inspired self-similar beam lattices, Applied Physics Letters 107 (22). doi:http://dx.doi.org/10.1063/1.4936564.

URL http://scitation.aip.org/content/aip/journal/apl/107/22/10.1063/1.4936564

[33] O. Sigmund, Materials with prescribed constitutive parameters: An inverse homogenization problem, International Journal of Solids and Structures 31 (17) (1994) 2313 - 2329. 
doi:http://dx.doi.org/10.1016/0020-7683(94)90154-6.

URL http://www.sciencedirect.com/science/article/pii/0020768394901546

[34] A. Slann, W. White, F. Scarpa, K. Boba, I. Farrow, Cellular plates with auxetic rectangular perforations, Physica status solidi (b) 252 (7) (2015) $1533-1539$.

[35] R. Lopes, N. Betrouni, Fractal and multifractal analysis: A review, Medical Image Analysis 13 (2009) 634-649.

[36] J. Feng, W.-C. Lin, C.-T. Chen, Fractional box-counting approach to fractal dimension estimation, in: Pattern Recognition, 1996. Proceedings of the 13th International Conference, IEEE, 1996, p. 854.

[37] M. M. Murr, D. E. Morse, Fractal intermediates in the self-assembly of silicatein filaments, Proceedings of the National Academy of Sciences 102 (33) (2005) 11657-11662.

[38] A. Costa, Hausdorff (box-counting) fractal dimension.

[39] A. Bezazi, F. Scarpa, C. Remillat, A novel centresymmetric honeycomb composite structure, Composite Structures 71 (3-4) (2005) 356 - 364. doi:10.1016/j.compstruct.2005.09.035.

URL http://www.sciencedirect.com/science/article/pii/S026382230500262X

[40] V. Salit, T. Weller, On the feasibility of introducing auxetic behavior into thin-walled structures, Acta Materialia 57 (1) (2009) 125 - 135.

[41] D. Prall, R. Lakes, Properties of a chiral honeycomb with a poisson's 
ratio of -1, International Journal of Mechanical Sciences 39 (3) (1997) 305-314. doi:10.1016/S0020-7403(96)00025-2.

[42] A. Bacigalupo, L. Gambarotta, Homogenization of periodic hexa- and tetrachiral cellular solids, Composite Structures 116 (0) (2014) 461-476. doi:http://dx.doi.org/10.1016/j.compstruct.2014.05.033.

URL http://www.sciencedirect.com/science/article/pii/S0263822314002438

[43] M. Collet, M. Ouisse, M. Ruzzene, M. Ichchou, Floquet-bloch decomposition for the computation of dispersion of two-dimensional periodic, damped mechanical systems, International Journal of Solids and Structures 48 (20) (2011) 2837-2848.

[44] J. Joannopoulos, S. Johnson, J. Winn, R. Meade, Photonic crystals: molding the flow of light, Princeton university press, 2011.

[45] R. Craster, T. Antonakakis, M. Makwana, S. Guenneau, Dangers of using the edges of the brillouin zone, Physical Review B 86 (11) (2012) 115130.

[46] L. J. Gibson, M. F. Ashby, Cellular solids: Structure and properties, 2nd Edition, Cambridge: Cambridge University Press, 1997.

[47] S. Gonella, M. Ruzzene, Homogenization and equivalent in-plane properties of two-dimensional periodic lattices, International Journal of Solids and Structures $45 \quad$ (10) (2008) $2897 \quad$ - 2915. doi:10.1016/j.ijsolstr.2008.01.002. 